\author{
Bruno Eckhardt
}

Editor

\title{
Advances in Turbulence
} XII

Proceedings of the 12th EUROMECH

European Turbulence Conference, September 7-10, 2009, Marburg, Germany

With 594Figures

Springer 
Professor Dr. Bruno Eckhardt

Philipps-Universität Marburg

Fachbereich Physik

Renthof 6

35032 Marburg, Germany

E-mail: bruno.eckhardt@physik.uni-marburg.de

ISSN 0930-8989

ISBN 978-3-642-03084-0

DOI 10.1007/978-3-642-03085-7

Springer Dordrecht Heidelberg London New York
e-ISSN 1867-4941

e-ISBN 978-3-642-03085-7

Library of Congress Control Number: 2009931716

(c) Springer-Verlag Berlin Heidelberg 2009

This work is subject to copyright. All rights are reserved, whether the whole or part of the material is concerned, specifically the rights of translation, reprinting, reuse of illustrations, recitation, broadcasting, reproduction on microfilm or in any other way, and storage in data banks. Duplication of this publication or parts thereof is permitted only under the provisions of the German Copyright Law of September 9, 1965 , in its current version, and permission for use must always be obtained from Springer-Verlag. Violations are liable to prosecution under the German Copyright Law.

The use of general descriptive names, registered names, trademarks, etc. in this publication does not imply, even in the absence of a specific statement, that such names are exempt from the relevant protective laws and regulations and therefore free for general use.

Cover design: SPi Publisher Services

Printed on acid-free paper

Springer is part of Springer Science+Business Media (www.springer.com) 


\title{
Universality of Kolmogorov law in spectrally condensed turbulence in thin layers
}

\author{
H. Xia, M.G. Shats, and H. Punzmann
}

Research School of Physics \& Engineering, The Australian National University, Canberra, ACT 0200, Australia

hua.xia@anu.edu. au

The four-fifth Kolmogorov law relates the third-order structure function and the energy dissipation rate $\epsilon$ in three-dimensional turbulence (see, e.g. [1]. This law for 2D turbulence reads: $S_{L 3}=(3 / 2) \epsilon r$, where $S_{L 3}=<\delta v_{L}^{3}>$ is the third moment of the longitudinal velocity increments at the scale $r$. Angular brackets denote averaging over the box size and time. This relationship allows the energy dissipation rate, or the energy flux within the inertial range to be determined by analyzing the third-order velocity moments in laboratory experiments or in observational data. The Kolmogorov law has been confirmed in direct numerical simulations of $2 \mathrm{D}$ turbulence [2], however previous experimental attempts to derive $\epsilon$ from the third-order longitudinal structure functions were not successful [3], probably due the insufficient statistics in the calculations of $\left\langle\delta v_{L}^{3}\right\rangle$. In this paper we present the results of the $S_{3}(r)$ measurements in quasi-2D turbulence in laboratory experiments. We are particularly interested in estimating the energy flux in the energy inertial interval and in comparison of its value with independently obtained energy dissipation rate.

Another practically interesting question is how the presence of the selfgenerated coherent flows in 2D turbulence affects the Kolmogorov law in the regime of spectral condensation. We investigate this in the thin stratified layers of fluid where turbulence is driven electromagnetically as described in $[4,5]$. When the flow is bounded the inverse energy cascade leads to the accumulation of the spectral energy at the scale close to the boundary size and generation of mean flow. By controlling the bottom dissipation rate (via the thickness of the fluid layers) and the size of the boundary, large vortices of different strengths, or condensates, can be obtained as discussed in [5].

Figure 1 shows time-averaged (over about 100s) velocity fields measured with the boundary size of $L=0.11 \mathrm{~m}$ for 3 different damping rates. The largescale vortex becomes more symmetric as its turnover time becomes shorter and approaches the inverse damping rate $1 / \alpha$. 


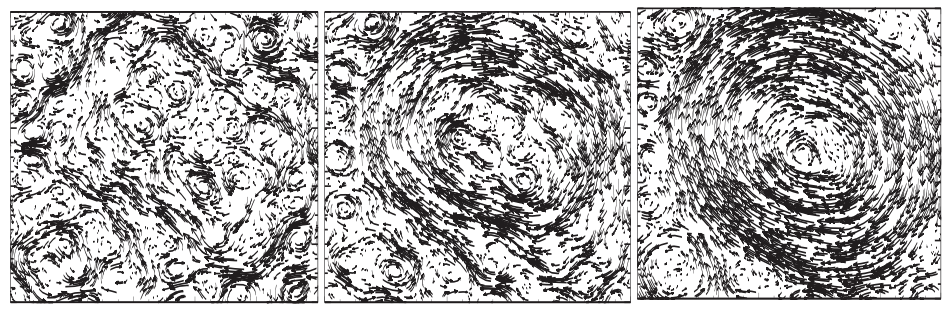

(a)

(b)

(c)

Fig. 1. Time-averaged velocity field of the condensate at $L \approx 0.10 \mathrm{~m}$ with different linear damping rate: (a) $\alpha=0.25 \mathrm{~s}^{-1}$, (b) $\alpha=0.15 \mathrm{~s}^{-1}$ and (c) $\alpha=0.05 \mathrm{~s}^{-1}$.

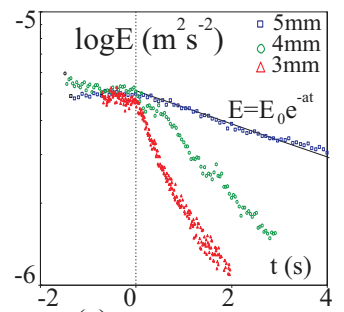

(a)

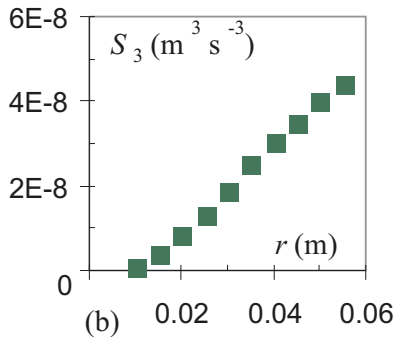

(b) $\quad 0.02 \quad 0.04 \quad 0.06$

Fig. 2. (a) Time evolution of the kinetic energy during turbulence decay for the 3 cases of Fig. 1. (b) 3rd-order structure function computed for the case of Fig. 1(c) by subtracting mean flow velocity.

The presence of the mean flow greatly affects the velocity moments, as discussed in [5]. However by subtracting mean (time-averaged) velocity field from instantaneous fields before computing the 3rd-order structure function $S_{3}=\left(S_{3 L}+S_{3 T}\right) / 2$ one recovers physically meaningfully values of $\epsilon=S_{3} / r$. This is verified by comparing $\epsilon$ with the energy dissipation rate derived from the time evolution of the turbulence energy in the initial stage and during the decay: $d E / d t-\alpha E=\epsilon$. During the decay $\epsilon \approx \alpha E$, while at the early stage of the turbulence development $d E / d t \approx \epsilon$. This result has been confirmed at different strengths of the condensate flow, even in the regimes when the presence of the condensate leads to the onset of non-Gaussian statistics in condensed turbulence (larger flatness).

\section{References}

1. U.Frisch, Turbulence: The Legacy of A.N. Kolmogorov (Cambridge, 1995).

2. G. Boffetta, A. Celani and M. Vergassola, Phys. Rev. E 61, R29, (2000).

3. J. Paret and P. Tabeling, Phys. Fluids 10, 3126, (1998).

4. M.G. Shats et al., Phys. Rev. Lett. 99, 164502, (2007).

5. H. Xia et al.,Phys. Rev. Lett. 101,194504, (2008). 